\title{
Handling incidental findings in neuroimaging research in Japan: current state of research facilities and attitudes of investigators and the general population
}

Misao Fujita ${ }^{1 *}$, Yoshinori Hayashi ${ }^{2}$, Shimon Tashiro ${ }^{3}$, Kyoko Takashima $^{4}$, Eisuke Nakazawa ${ }^{4}$ and Akira Akabayashi ${ }^{4}$

\begin{abstract}
Background: To establish appropriate measures that deal with incidental findings (IFs), the neuroscience community needs to address various ethical issues. The current state of research facilities regarding IFs and investigator attitudes as well as potentially eligible research participants must be assessed prior to future discussions and before the development of policies and guidelines. To this end, we conducted two questionnaire surveys to clarify i) how IFs are addressed at neuroimaging research facilities in Japan and ii) the views of investigators and potential research participants regarding the handling of IFs.
\end{abstract}

Methods: Thirty-one principal investigators (PIs) involved in the Strategic Research Program for Brain Sciences (SRPBS), a government-funded project, were asked to fill out a questionnaire regarding ways IFs were handled at the facility. A total of 110 investigators engaged in SRPBS tasks, including 31 Pls who participated in the research facility survey and researchers conducting studies under the management of the Pls, and 500 individuals from the general public (i.e., general population) were asked to select the most appropriate way to deal with IFs in two scenarios, namely the medical school and humanities and social sciences department scenarios.

Results: More than 40\% of Pls responded that they did not know or were unsure of what type of approach was employed to handle IFs at their research facilities. Nevertheless, they were willing to improve the current status if sufficient resources were provided. With regard to specialist involvement, 37.7\% of investigators responded that it was appropriate to have a specialist check all images in the medical school scenario, whereas $13.3 \%$ responded that such involvement was appropriate in the humanities and social sciences department scenario. In contrast, $76.1 \%$ and $61.0 \%$ of the general population indicated that specialist involvement was appropriate in the medical school and humanities and social sciences department scenarios, respectively. These results show that expectations of the general population exceed those of investigators regarding measures to address IFs. Both investigators and the general population demanded more responsibility from Pls at medical institutions, compared to Pls at non-medical institutions.

Conclusions: Based on our preliminary results, we recommended that a licensed physician perform a screening test to appropriately examine clear abnormalities. These recommendations were implemented by the SRPBS as guidelines for handling IFs in national research projects in Japan.

Keywords: General population, Incidental findings, Investigators, Japan, Neuroimaging, Questionnaires

\footnotetext{
* Correspondence: misao-fujita@cira.kyoto-u.ac.jp

'Uehiro Research Division for iPS Cell Ethics, Center for iPS Cell Research and Application (CiRA), Kyoto University, The Kyoto Technoscience Center \#3, 14, Yoshidakawara-cho, Sakyo-ku, Kyoto 606-8305, Japan

Full list of author information is available at the end of the article
} 


\section{Background}

An incidental finding (IF) is defined as "a finding concerning an individual research participant that has potential health or reproductive importance and is discovered in the course of conducting research but is beyond the aims of the study" [1]. In neuroimaging research, IFs include brain tumors, cerebral aneurysms, or asymptomatic vascular lesions, which are not directly related to research aims but are found by magnetic resonance imaging (MRI) or research MRI sequences. One study reported that the frequency of brain-related IFs that are found by MRI or functional magnetic resonance imaging (fMRI) studies is $13 \%$ to $84 \%$ [1]. In that study, $1.2 \%$ of findings required immediate referral for clinical evaluation, whereas $13 \%$ to $40.4 \%$ of findings did not [1]. Recently, the neuroscience research community has aimed to establish measures to handle such findings, as various ethical questions regarding IFs have been raised [2].

Research MRI images typically have lower resolution and contrast [3], and are often unsuitable for clinical diagnoses. Moreover, opportunities for non-physician investigators who are untrained in image evaluation to conduct neuroimaging research in non-medical areas (e.g., cognitive psychology and behavioral economics) have increased. This can lead to an increased risk of false-negative errors (i.e., a disease is overlooked) and false-positive errors (i.e., a disease is inaccurately diagnosed). False-negative errors can lead a participant to ignore the early symptoms of a disease and thereby aggravate his or her condition. In fact, the majority of healthy adults who participate in neuroimaging studies expect the study to discover any abnormality that exists [4]. In contrast, false-positive errors might significantly burden participants (i.e., mentally, economically, and temporally) until the absence of disease is confirmed. Indeed, one study revealed that subsequent examination confirmed all detected IFs to be false positives [5].

There is a general consensus within the research community that IFs should be addressed appropriately [6,7]. However, there exists evidence that IFs are handled differently across research facilities. According to a survey conducted by Lawrenz and Sobotka, informed consent forms that mentioned IFs were used in approximately 9\%, 25\%, $11 \%$, and $37 \%$ of cases at federal institutions, relevant conferences, universities, and other facilities, respectively, and the contents differed for each form [8]. An Internet survey conducted by Illes et al. showed that more than $80 \%$ of investigators encountered IFs during their studies; however, only half of these investigators indicated that a standard procedure existed for addressing IFs at their research facilities [9]. With respect to specialist (neuroradiologist) involvement, their responses ranged from "checks all images" and "checks only equivocal findings" to "checks no images at all" [9].
Opinions regarding appropriate measures to be taken by principal investigators (PIs) are also divided. Royal and Peterson are against specialist involvement, as they believe such involvement may increase the risk of falsepositive errors. In other words, a specialist, who is concerned "about medicolegal liability from failing to identify a serious IF," may "err on the 'safe side' by recommending further consultation" [10]. According to Richardson, actively searching for abnormal findings violates participant privacy, although the PI is responsible for providing "ancillary care" for a disease discovered during a study [11]. Another view suggests that the reading of all images or use of clinical scans by specialists is not always required [2,12]. In contrast, Milstein asserts that the PI should provide the highest level of care (e.g., detailed examinations and the use of clinical devices), because otherwise, $\mathrm{s} / \mathrm{he}$ may be sued if a disease worsens or the participant develops a disorder during the course of research [13].

Given that various approaches are adopted by research facilities with differing opinions among scholars, the recommendations summarized during the Detection and Disclosure of Incidental Findings in Neuroimaging Research workshop, which was held in 2005 by the National Institutes of Health (NIH) and Stanford University, represented a significant breakthrough [14]. Five options were provided for addressing IFs so that investigators can implement the most appropriate option for them. In 2008, Wolf et al. provided more detailed recommendations regarding the ethical and legal aspects of IFs in neuroimaging and genetic/genomic research [1]. Furthermore, in October 2012, the NIH and a working group supported by US and Canadian government agencies discussed measures to address IFs in neuroimaging research, and published the results online $[15,16]$. However, directly adopting these recommendations in countries other than the US and Canada would require careful consideration.

In Japan, the Ministry of Education, Culture, Sports, Science, and Technology (MEXT) launched the Strategic Research Program for Brain Sciences (SRPBS) in 2008 to strategically advance neuroscience research [17]. Government research conducted with public funds includes many neuroimaging studies that use MRI and fMRI techniques. However, the only guidance available regarding IFs in neuroimaging research is found in the Guidelines on Ethical Issues of Noninvasive Research on Human Brain Function, which was revised in 2009 by the Japanese Neuroscience Society [18]. These guidelines deem it appropriate to consult a specialist prior to disclosing an IF to a participant, but the reasons for this approach are not mentioned. Moreover, these guidelines only have binding power on association members. In general, there has been little discussion regarding how IFs should be addressed in Japan $[19,20]$. 
To facilitate future discussions and develop additional policies or guidelines, we must first assess the current state of relevant research facilities, and clarify attitudes of investigators and potentially eligible research participants regarding IFs. However, few empirical studies have investigated the actual procedures for addressing IFs at research facilities in Japan $[19,20]$. Moreover, the focus has been placed on conducting surveys of investigators in most international studies $[9,21]$, or of participants who have already taken part in research [4,22,23], and few studies have investigated the attitudes of potential research participants regarding IFs procedures. Thus, whether their attitudes differ from those of investigators is unclear. Therefore, the present study used two questionnaires to survey the following: i) how IFs are addressed at neuroimaging research facilities in Japan, and ii) the views of investigators and potential research participants regarding the handling of IFs. As issues surrounding IFs are still somewhat unexplored in Japan, we used a modified version of the five options established by Illes et al. [14], along with two scenarios, to help participants better understand the questionnaires.

\section{Methods}

Research participants

Research facility survey

PIs of 31 research facilities involved in the SRPBS between 2008 and 2010 were recruited for this survey. These research facilities were engaged in one of the following tasks: "Development of brain-machine interfaces," "Development of highly original animal models," and "Development of technologies to measure and assist brain mechanisms that support social behaviors." The questionnaires were mailed according to the address of each research facility and the names of corresponding PIs listed on the SRPBS website [17].

\section{Investigators/general population survey}

Study participants were a total of 110 investigators engaged in SRPBS tasks, including 31 PIs who participated in the research facility survey and researchers conducting studies under the management of the PIs. A list of their addresses and names was obtained, and the questionnaires mailed, upon approval of the SRPBS. The general population in this survey refers to registered members of the Japan Management Association Research Institute, which recruits volunteers who participate in various studies [24]. A questionnaire was uploaded onto the institute's website, and registered volunteers were able to choose whether or not to answer the questionnaire. The questionnaire remained available until 500 responses were received. We collected 100 from each age group ranging from 20 to 60 years (250 from each gender) to avoid sampling biases.

\section{Questionnaires}

Between October and November 2010, the PIs, investigators, and the general population were asked to complete an anonymous self-administered questionnaire. IFs were defined as follows:

\section{“'Incidental findings (IFs)' refer to unexpected health problems (e.g., brain tumors, cerebral aneurysms, or asymptomatic vascular disorders) discovered by coincidence during a study on a research participant's MRI and/or fMRI brain images. The frequency of IFs has been reported to be 13-84\%, and 1.2\% of IFs require immediate examination. Study participants benefit from these IFs because they are made aware of their disease.}

However, imaging devices used for research purposes may result in false-negative (i.e., judged healthy despite having a disease) or false-positive (i.e., judged to have a disease despite being healthy) errors. In falsepositive cases, participants may experience unnecessary stress and economic burden until the absence of disease is confirmed."

The survey respondents were also informed that MRI is an examination method that uses a computer to render images of cross-sections of the body and that fMRI visualizes brain activity using MRI. It took about 5 to $10 \mathrm{~min}$ to complete the questionnaires. Only the general population received a reward in the form of points (approximately 20 to 30 points, which are exchangeable for money at a rate of 1 yen/point) from the research firm. This system is widely implemented by research firms in Japan.

\section{Research facility survey}

In the questionnaire, we asked PIs i) questions regarding basic characteristics, ii) how IFs are addressed at the research facility ("At the research institution to which you belong, which of options 1-6 (Table 1) is currently employed as a means of addressing IFs?"), and iii) what would be the appropriate way to address IFs if sufficient resources were available ("If the research institution to which you belong had adequate resources (e.g., personnel, equipment, and research funds) to address IFs, which of options 1-6 do you think would be most appropriate in terms of the extent of the response?"). These six options were obtained by reorganizing the five options established by Illes et al. [14] to facilitate statistical analyses.

\section{Investigators/general population survey}

The questionnaire included i) basic characteristic questions, ii) a scenario of an abundantly funded neuroimaging research study of patients at a medical school, which was 


\begin{tabular}{|c|c|}
\hline Option 1 & $\begin{array}{l}\text { When obtaining informed consent }(I C) \text {, the } \\
\text { possibility of IFs is not explained at all to the participant. }\end{array}$ \\
\hline Option 2 & $\begin{array}{l}\text { When obtaining IC, the participant is informed that "IFs } \\
\text { may be discovered, but will not be explained even if } \\
\text { they are found." }\end{array}$ \\
\hline Option 3 & $\begin{array}{l}\text { When obtaining IC, the participant is informed that "IFs } \\
\text { may be discovered, and if the principal investigator (PI) } \\
\text { considers them suspect, s/he will notify the participant. } \\
\text { However, a specialist (radiologist) does not check the } \\
\text { images." }\end{array}$ \\
\hline Option 4 & $\begin{array}{l}\text { When obtaining IC, the participant is informed that "if IFs } \\
\text { are suspected, a specialist (radiologist) will be asked to } \\
\text { check the images. If a genuine problem appears to exist, } \\
\text { the PI will notify the participant." The cost for specialist } \\
\text { consultation is obtained from the PI's research funds. }\end{array}$ \\
\hline Option 5 & $\begin{array}{l}\text { When obtaining IC, the participant is informed that "a } \\
\text { specialist (radiologist) will check all images to discover } \\
\text { IFs. If a genuine problem appears to exist, the PI will } \\
\text { notify the participant." The cost for specialist consultation } \\
\text { is obtained from the PI's research funds. }\end{array}$ \\
\hline Option 6 & $\begin{array}{l}\text { When obtaining IC, the participant is informed that "a } \\
\text { clinical device with more precision than that used for } \\
\text { research will be initially used to detect IFs. A specialist } \\
\text { (radiologist) will check all images, and the PI will notify } \\
\text { the participant when a firm diagnosis is established." The } \\
\text { costs for the device and specialist consultation are } \\
\text { obtained from the PI's research funds. }\end{array}$ \\
\hline
\end{tabular}

conducted by a PI with a physician's license (Medical school scenario), and iii) a scenario of a poorly funded neuroimaging research study of healthy individuals in a humanities or social sciences department, which was conducted by a PI without a physician's license (Humanities and social sciences department scenario). We employed these two scenarios based on a previous study showing that attitudes of research participants toward IFs vary depending on whether the research context is medical or non-medical [4]. For ii) and iii), we asked all respondents to select the most appropriate way to address IFs from the six options. In addition, following Kirschen et al. [4], we asked the general population about their iv) expectations regarding image assessment in neuroimaging research.

\section{Medical school scenario}

A PI who is employed at a medical school-affiliated university hospital is planning a study to clarify the relationship between a person's emotions and neural activity. Fifty patients commuting to the affiliated hospital (who are at least 20 years old) are recruited as participants. To measure neural activity, the plan is to use an fMRI device that has a lower precision than does a device that would be used in the clinical context. The PI is a licensed physician who has previously conducted numerous studies using fMRI devices. The allowable expenditure for this study is 10 million yen per year. What approach should the PI adopt to address IFs?

\section{Humanities and social sciences department scenario}

A PI who is employed at a humanities and social sciences department of a university is planning a study to clarify the relationship between a person's emotions and neural activity. Fifty healthy students from the university (who are at least 20 years old) are recruited as participants. To measure neural activity, the plan is to use an fMRI device that has a lower precision than does a device that would be used in the clinical context. Although not a licensed physician, the PI has previously conducted numerous studies using fMRI devices. The allowable expenditure for this study is 1 million yen per year. What approach should the PI adopt to address IFs?

\section{Statistical analyses}

We performed Wilcoxon rank sum and signed rank sum tests using SAS version 9.1. To test for significance, we used a two-tailed test with a criterion level of $5 \%$.

\section{Ethical considerations}

This study was approved by the Ethics Committee of the Tokyo University Graduate School of Medicine and Faculty of Medicine.

\section{Results}

\section{Research facility survey}

We mailed questionnaires to 31 PIs and obtained responses from 23 (response rate, 74.1\%; Table 2). Of the 14 PIs who were conducting human research using imaging devices at their research facilities, 6 (42.9\%) reported prior experience with IFs. These findings included brain tumors, cerebrovascular disorders, cerebral aneurysms, arachnoid cysts, and sinusitis. Figure 1 shows the current and ideal approaches for handling IFs according to the answers provided by the 14 PIs. Six (42.9\%) answered that they were unsure of whether institutional guidelines exist, and 5 (35.7\%) selected option 3 (Table 1) as the current institutional policy. However, half of the PIs indicated that specialist involvement would be appropriate (option 4 or higher) if resources were available. None of them currently adopted option 1 or option 2, or considered these options ideal. A comparison of responses concerning the current approach and those concerning the ideal approach using the Wilcoxon signed rank sum test revealed a significant difference $(P=0.0156)$.

\section{Investigators/general population survey}

Of the 110 investigators to whom questionnaires were mailed, 70 responded (response rate, 63.6\%; Table 2). Of the 33 investigators who were conducting human research, 10 (30.3\%) reported having experience with IFs. These findings included brain tumors, normal pressure hydrocephalus, lacunar infarctions, venous malformations, cavernous hemangiomas, cerebral aneurysms, arachnoid 
Table 2 Basic characteristics

\begin{tabular}{|c|c|c|c|c|}
\hline & & $\begin{array}{l}\text { Pls } \\
\mathrm{n}=23 \text { (\%) }\end{array}$ & $\begin{array}{l}\text { Investigators } \\
\mathrm{n}=69^{\mathrm{b}}(\%)\end{array}$ & $\begin{array}{l}\text { General population } \\
\mathrm{n}=500(\%)\end{array}$ \\
\hline \multirow[t]{2}{*}{ Sex } & Male & $22(95.7)$ & $65(94.2)$ & $250(50.0)$ \\
\hline & Female & $1(4.4)$ & $4(5.8)$ & $250(50.0)$ \\
\hline \multirow[t]{5}{*}{ Age range (years) } & $20-29$ & $0(0)$ & $0(0)$ & $100(20.0)$ \\
\hline & $30-39$ & $2(8.7)$ & $14(20.3)$ & $100(20.0)$ \\
\hline & $40-49$ & $10(43.5)$ & $25(36.2)$ & $100(20.0)$ \\
\hline & $50-59$ & $8(34.8)$ & $25(36.2)$ & $100(20.0)$ \\
\hline & $>59$ & $3(13.0)$ & $5(7.3)$ & $100(20.0)$ \\
\hline \multirow[t]{6}{*}{ Affiliation } & University medical school & $10(43.5)$ & $31(45.6)$ & \\
\hline & University science and engineering department & $2(8.7)$ & $10(14.7)$ & \\
\hline & University humanities and social sciences department & $1(4.4)$ & $2(2.9)$ & \\
\hline & Non-university research institution & $4(17.4)$ & $13(19.1)$ & \\
\hline & Company & $3(13.0)$ & $4(5.9)$ & \\
\hline & Other & $3(13.0)$ & $8(11.8)$ & \\
\hline \multirow[t]{2}{*}{ Licensed physician } & Yes & $10(43.5)$ & $35(50.7)$ & \\
\hline & No & $13(56.5)$ & $34(49.3)$ & \\
\hline \multirow[t]{2}{*}{ Use of neuroimaging devices } & Yes & $16(69.6)$ & $26(37.7)$ & \\
\hline & No & $7(30.4)$ & $43(62.3)$ & \\
\hline \multirow[t]{6}{*}{ Research subjects } & Non-human & $8(34.8)$ & $34(50.0)^{c}$ & \\
\hline & Human & $15(65.2)$ & $34(50.0)^{c}$ & \\
\hline & w/ patients & $12(80.0)$ & $25(75.8)^{d}$ & \\
\hline & w/o patients & $3(20.0)$ & $8(24.2)^{d}$ & \\
\hline & W/ IF experience & $6(42.9)^{a}$ & $10(30.3)^{d}$ & \\
\hline & W/o IF experience & $8(57.1)^{a}$ & $23(69.7)^{d}$ & \\
\hline \multirow[t]{3}{*}{ Guidelines on IFs available } & Yes & $4(18.2)$ & $8(11.6)$ & \\
\hline & No & $12(54.6)$ & $27(39.1)$ & \\
\hline & Unsure & $6(27.3)$ & $34(49.3)$ & \\
\hline \multirow[t]{2}{*}{ Experience with tests that employ neuroimaging technology } & Yes & & & $145(29.0)$ \\
\hline & No & & & $355(71.0)$ \\
\hline
\end{tabular}




\section{Table 2 Basic characteristics (Continued)}

Experience participating in studies that use neuroimaging technology

$$
\text { Yes }
$$

W/ IF experience

No

${ }^{\mathrm{a}}$ Of the 15 Pls who were conducting research on humans, one did not respond and was thus excluded from the calculations. b Of the 70 investigators, one with incomplete information was excluded from the calculations.

'Of the 69 investigators with complete information, one without responses was excluded from the calculations.

${ }^{\mathrm{d}}$ Of the 34 investigators who were conducting research on humans, one without responses was excluded from the calculations. 


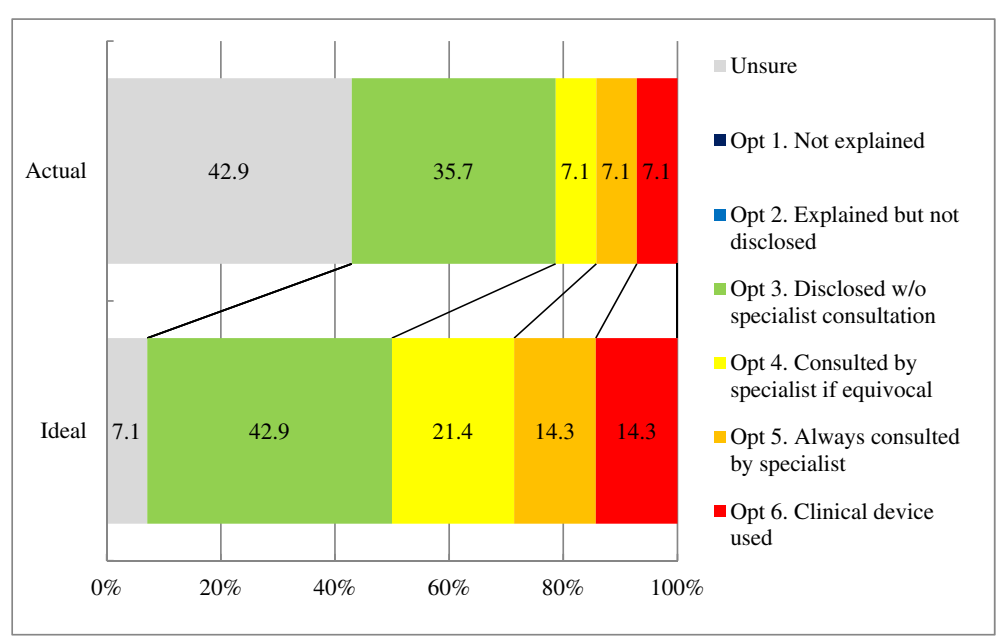

Figure 1 Comparison of actual and ideal treatments of incidental findings. Calculations were based on data obtained from the 14 principal investigators who conduct human research using imaging devices.

cysts, carotid artery malformations, and sinusitis. Of the 500 individuals from the general population who answered the web-based questionnaire, $8(1.6 \%)$ had prior experience participating in research using neuroimaging technology. Of these, 2 were found to have central nervous system vasculitis and cerebral infarction during the course of study participation.

\section{Medical school scenario}

More than 97\% (67/69) of investigators considered it appropriate to notify the participant if IFs were discovered (option 3 or higher; Figure 2). However, 62.3\% (43/69) of investigators considered it unnecessary to have a specialist check the images (option 3 or lower), whereas 37.7\% indicated that having a specialist check the images was desirable (26/69; option 4 or higher). Only 4.4\% (3/69) of investigators responded that it was desirable to have a specialist check all images (option 5 or higher), and none supported the use of more precise clinical devices for the purpose of detecting IFs (option 6). The Wilcoxon rank sum test revealed that the responses did not differ significantly according to whether an investigator was a licensed physician, was affiliated with a medical school, had used imaging equipment, had conducted human research, or had guidelines for addressing IFs.

In contrast, only $23.8 \%(119 / 500)$ of the general population considered it unnecessary to have a specialist check the images (option 3 or lower), whereas 76.1\% (381/500) indicated that having a specialist check the images (option 4 or higher) was desirable. The proportion of individuals who responded that it was desirable to have a specialist check all images (option 5 or higher) was 38.9\% (195/500),

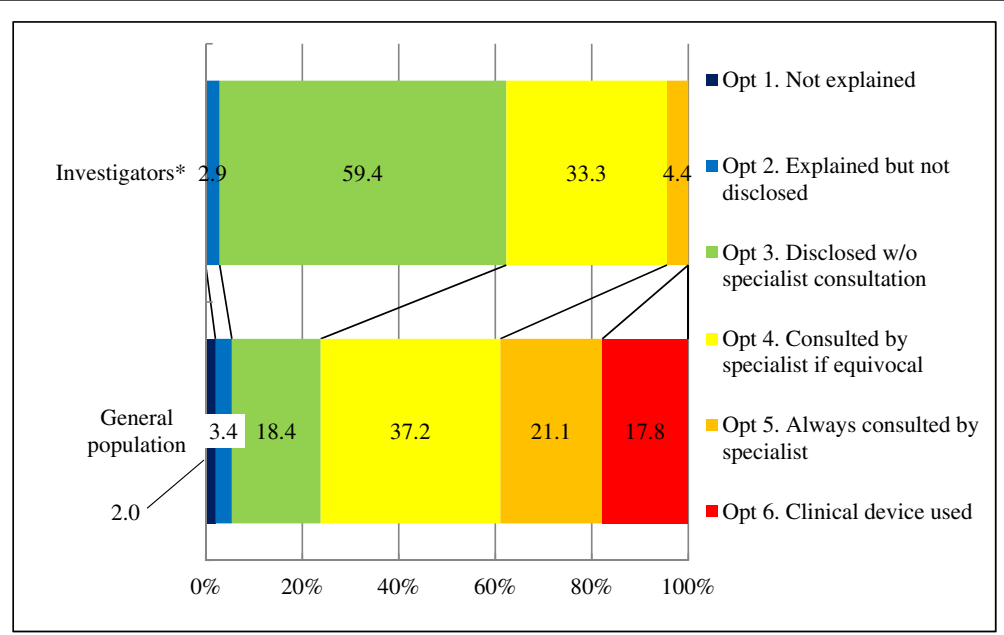

Figure 2 Appropriate approaches that principal investigators should adopt according to the medical school scenario. ${ }^{*}$ Of the 70 investigators, one without complete information was excluded from the calculations. 
and that of those who supported the use of clinical devices (option 6) was 17.8\% (89/500). A significant difference was detected $(P<0.0001$; Wilcoxon rank sum test) in responses between investigators and the general population.

\section{Humanities and social sciences department scenario}

Approximately 75\% (50/68) of investigators considered it important to notify the participant if IFs were discovered (option 3 or higher; Figure 3). However, 86.8\% (59/68) of investigators considered it unnecessary to consult a specialist (option 3 or lower). Only 13.3\% (9/68) of investigators indicated that a specialist should check the images (option 4 or higher), and none supported the use of clinical devices (option 6). The Wilcoxon rank sum test revealed that responses did not differ significantly according to whether an investigator was a licensed physician, was affiliated with a medical school, had used imaging equipment, had conducted human research, or had guidelines for handling IFs.

Among the general population, 39.0\% (195/500) considered specialist consultation unnecessary (option 3 or lower), whereas $61.0 \%(305 / 500)$ responded that it was desirable (option 4 or higher). Of these, approximately half $(31.6 \%)$ indicated that a specialist should check all images (option 5 or higher), and 15\% (75/500) supported the use of clinical devices (option 6). A significant difference was detected $(P<0.0001$; Wilcoxon rank sum test $)$ in responses between investigators and the general population.

\section{Comparison of the medical school and humanities and social sciences department scenarios}

A significant difference $(P<0.0001$; Wilcoxon signed rank sum test) was observed when investigator responses were compared between the medical school and humanities and social sciences department scenarios. Similarly, a significant difference was observed in responses among the general population $(P<0.0001)$.

\section{General population's view on handling IFs}

In response to the question, "How do you think investigators verify whether a participant has an abnormality during a research study using MRI or fMRI," $80.8 \%$ $(404 / 500)$ of the general population indicated that they expected specialist involvement (Table 3).

\section{Discussion}

This study is the first to assess the procedures for addressing IFs in neuroscience research facilities in Japan. Unlike previous studies [4,9,21-23], we compared the views of investigators and the general population regarding the handling of IFs. Moreover, the policy regarding IFs was implemented by the SRPBS, which took into consideration the preliminary results of this study.

\section{Current state of neuroimaging research facilities}

Of the 14 research facilities that conduct neuroimaging research on humans, $40 \%$ had previously encountered IFs. Nonetheless, more than $40 \%$ of PIs indicated that they did not know or were unsure of what type of approach was employed at their facilities. This finding is consistent with previous reports on research facilities in many other countries, and not unique to Japanese facilities. A previous study found that $82 \%$ of investigators reporting MRI-related studies had experienced IFs during their studies; only 53\% indicated that there was a standardized facility procedure to address such discoveries [9]. In a survey of investigators from a leading Canadian neuroimaging center, the investigators emphasized

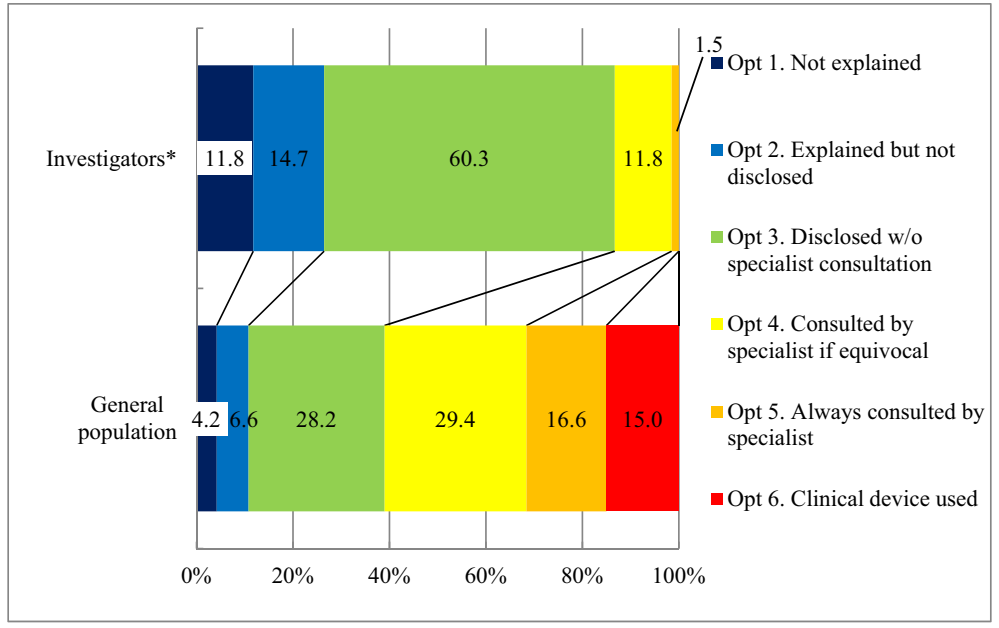

Figure 3 Appropriate approaches that principal investigators should adopt according to the humanities and social sciences department scenario. * Of the 70 investigators, two without complete information were excluded from the calculations. 


\begin{tabular}{|c|c|}
\hline Modes of evaluation & n (\%) \\
\hline No one checks the images & $41(8.2)$ \\
\hline PI checks the images & $55(11.0)$ \\
\hline $\begin{array}{l}\text { Specialist checks the images only when there is a } \\
\text { suspected finding }\end{array}$ & $294(58.8)$ \\
\hline Specialist checks all images & $90(18.0)$ \\
\hline Specialist uses a clinical device to check all images & $20(4.0)$ \\
\hline
\end{tabular}

that there were no procedures or guidelines in place to address IFs [21].

In the present study, more than $90 \%$ of PIs indicated that option 3 or higher was desirable if sufficient resources were available, suggesting that the majority of PIs considered it appropriate to provide explanations of IFs to participants in advance and to notify them when such findings are discovered. Moreover, the gap between the actual and ideal approaches to IFs implied that PIs were willing to improve the handling of IFs, as long as sufficient resources are provided. When the government funds these facilities, they should subsidize the expenses required to establish policies and guidelines regarding IFs and to recruit specialists for image evaluation to improve IF-related efforts.

\section{Preferences regarding specialist involvement}

Views of investigators and the general population regarding specialist involvement differed considerably. The proportion of investigators who indicated that specialist involvement was desirable (option 4 or higher) was $37.7 \%$ in the medical school scenario and $13.3 \%$ in the humanities and social sciences department scenario. In contrast, $76.1 \%$ and $61.0 \%$ of the general population indicated that specialist involvement was desirable in the medical school and humanities and social sciences department scenarios, respectively (option 4 or higher). This discrepancy likely reflects the different views of PIs and the general population regarding the current state of research facilities. The PIs who responded to the questionnaire were already aware that specialists are not always involved in neuroimaging research on humans (Figure 1). In contrast, $80 \%$ of the general population expected specialists to check the images in research facilities (Table 3 ).

Illes and Chin stated that participants may suffer harm if no clinical evaluation is performed when there is a finding that urgently requires further examination [2]. Therefore, they concluded that it would be appropriate to entrust the image readings to a trained specialist [2]. Mamourian also supports this conclusion based on his personal experience after finding a cerebral aneurysm on his images during volunteer work in an MRI study [25]. Based on our findings, we can speculate that the general population expects specialist involvement as a means to avoid false-negative errors. However, Royal and Peterson believe that specialists are more likely to recommend more detailed examinations because they fear liability, causing mental, economical, and temporal burdens on participants with false-positive errors [10]. One potential reason for the difference in opinions reported across the literature is that there are insufficient data to determine how the involvement of a specialist would impact the risk of false-negative and false-positive errors or the participants' welfare. A challenge for future studies will be to empirically determine detection rates of IFs for specialists and PIs and compare them with subsequently confirmed disease incidence rates.

\section{Reactive responses vs. proactive responses}

Almost all investigators who indicated that specialist involvement was appropriate (option 4 or higher) considered a "reactive" response adequate, only when there was a suspicious finding (option 4). On the other hand, more than half of the general population considered a "proactive" [26] response appropriate, which involves a specialist routinely checking images and actively searching for IFs (option 5 or higher). Furthermore, half of the general population supported a "very proactive" [26] response, i.e., taking images with a clinical device that are not intended for research (option 6). In contrast, none of the investigators chose this option.

At first glance, "proactive" and "very proactive" response models seem superior to a "reactive" response model in terms of the ability to reduce disease oversight (i.e., risk of false-negative errors) by increasing the detection rate of abnormal findings. This approach is taken by a number of research facilities, including the $\mathrm{NIH}$ [3,25,27-29]. In addition, if a specialist joins a research team in advance and immediately checks all images, s/he could address any situations that require immediate or emergency follow-up [30], which, in turn, will benefit the participant.

However, it is an act that goes beyond formal research objectives to provide a benefit to participants. Therefore, a number of experts question the appropriateness of attempting to reduce the risk of false-negative errors $[11,12]$. In their view, the search for health problems that would ordinarily be overlooked deviates from research objectives and is closer to medical practice [11,12,31]. Even if such practices are regarded as responsibilities of a physician within a clinical setting, it is difficult to regard them as fundamental responsibilities of the PI [12]. Some believe that results counterbalancing the detection rate for clinically important IFs, believed to be $2 \%$ to $8 \%$ [14], are unrealistic to expect, and attempts to implement these models are impractical in light of the enormous effort and expenditure required $[3,14,30,32,33]$. Our results, which 
indicate that few investigators support the "proactive" and "very proactive" models, are consistent with the aforementioned views toward PIs' responsibilities and model feasibility.

We found that the general population tends to support measures that reflect expectations that exceed those of investigators (e.g., the introduction of therapeutic elements into research), suggesting the presence of a "therapeutic misconception" among the general population regarding research. In other words, the general public may erroneously expect therapeutic benefits from participating in a research study $[34,35]$. Consent to participate in a study based on misunderstandings such as expectation of benefits or underestimation of risk does not represent true informed consent $[1,7,11,12,26,36]$. As previously demonstrated by a number of neuroimaging studies $[4,22,23]$, the present study findings also indicate the tendency of the general public to expect practices similar to medical examinations; in this regard, no distinction is made between research and medical practice. A more detailed interview study targeting the general public and research participants will be required to confirm this finding.

\section{PI responsibilities under various conditions}

Both investigators and the general population supported a more rigorous response in the medical school scenario than in the humanities and social sciences department scenario. Approximately $40 \%$ of investigators indicated that specialist involvement would be appropriate (option 4 or higher) in the medical school scenario, as opposed to approximately $13 \%$ in the humanities and social sciences department scenario. In contrast, responses that included "no explanation of IFs" (option 1) and "no notification even if IFs were found" (option 2) increased 9-fold in the humanities and social sciences department scenario from $2.9 \%$ in the medical school scenario. These findings were unrelated to investigator background. Interestingly, a similar trend was observed in the general population.

Our results are consistent with the conclusions reported by Illes and Chin, and with the recommendations provided by the Detection and Disclosure of Incidental Findings in Neuroimaging Research workshop, which states that there is no single correct approach to IFs, and more than one morally acceptable option may exist depending on the research environment or specialization of the PI $[2,14]$. Milstein asserts that PIs may be legally required to provide measures that meet the highest standards of medical practice, regardless of differences in their research environments or specializations compared to those of medical doctors [13]. However, both investigators and the general population in our study demanded more responsibility from PIs with physician licenses at medical institutions, although they were more lenient toward nonlicensed PIs at non-medical institutions. As the present study did not provide any insight regarding the reason behind this outcome, further research is warranted.

\section{Recommendations for handling IFs according to the SRPBS}

Based on the relevant publications $[7,31]$ and our preliminary results, we submitted a recommendation [37] to the SRPBS suggesting the following approach for addressing IFs.

\section{With respect to all brain images taken during a neuroscience research study conducted by the SRPBS, it is desirable that a licensed physician performs a screening test to appropriately examine if a clear abnormality exists. For the time being, we will treat every abnormality this way on a trial basis to help shed light on unforeseen issues. Re-examination of this method after a year is desirable [37].}

Adopting option 1 or 2 , in which no action is taken in response to a finding, essentially denies the PI's responsibility to the participant. Even though a PI will not be held liable as a physician would, such behavior may be considered irresponsible, as the project is government funded. There is a general consensus among the PIs in our study (Figure 1) and the overseas research community regarding PI responsibility in addressing IFs [6,7].

In option 3 , in which a specialist is not involved, errors associated with false negatives and false positives are expected to increase [31,37]. Furthermore, the general population in our study considered specialist involvement appropriate regardless of the PI's specialization or research environment (Figures 2 and 3, and Table 3). The option without specialist involvement is unsuitable for research institutions receiving public funds, given that the majority of the general population desired a more aggressive response in view of the potential harm that can result from false-negative and false-positive errors.

If reducing errors (i.e., false negatives and false positives) is our goal, then option 4 is also inadequate. Although false-positive errors are likely to decrease if a discovered finding is confirmed by a specialist, falsenegative errors would still remain high in this setting, because the study is not performed by a specialist. Therefore, options that potentially reduce both types of errors (option 5 or higher) are more desirable. However, option 6 comes exceedingly close to medical practice, and may not only exacerbate "therapeutic misconception" by participants but also go beyond expected fundamental responsibilities of PIs. Although a small proportion of the general population selected option 6 (Figures 2 and 3) in our study, an option that exacerbates "therapeutic misconception" could distort risk- 
benefit calculations by research participants, making true informed consent impossible $[1,7,11,12,26,36]$.

For all brain images taken during the course of research studies conducted as part of the SRPBS, we conclude that image evaluation by a specialist is desirable (option 5). We also recommend that investigators be thorough in obtaining informed consent in an effort to discourage "therapeutic misconception" and that the SRPBS provide economic assistance to research facilities that may have difficulty implementing this option. These recommendations were adopted by the SRPBS and implemented on a trial basis in April 2012. However, consistent with the investigators' views in the present study (Figures 2 and 3), several studies do not support option $5[3,11,12,14,30,32,33]$. For this reason, future studies on investigator and participant attitudes and IF detection rates are required to evaluate the success of the implemented proposal. Investigation of the impact of IF-addressing measures based on the present proposal, as well as IF detection rates among all images in the SRPBS, is currently underway.

\section{Conclusions}

It is important to note that our study had some sampling limitations. First, study participants included a small number of PIs and investigators. Given that many of them belonged to leading Japanese research facilities with relatively abundant human and economic resources, other options may have been selected, if PIs and investigators from outside the SRPBS were included. Moreover, not all investigators conducted human research using MRI or fMRI. However, our statistical analyses revealed that the type of research or use of imaging equipment did not alter investigator attitudes toward IFs handling procedures.

Second, the general population consisted of individuals who volunteer to participate in studies conducted by a research firm, and who decided to complete our questionnaire. Therefore, we cannot rule out the possibility that the majority of these participants had an interest in neuroimaging research and that they did not truly represent the general public regarding views on IF issues. However, given the likelihood that our participants responded to the questionnaire without fully understanding what an IF is, it is possible that their views are similar to those held by potentially eligible research participants.

The questionnaires used in this study did not address issues of how or who should notify participants in the case of a suspicious IF, despite the relative importance of these issues. This could be considered another limitation. We are currently investigating disclosure conditions at each facility conducting an SRPBS project, as well as the impact of disclosing findings on research participants.

We conducted two questionnaire surveys to clarify i) how IFs are addressed at neuroimaging research facilities in Japan and ii) the views of investigators and the general population regarding the handling of IFs. We found that almost half of the PIs at facilities conducting human research using MRI or fMRI had no or little knowledge regarding the type of approach being employed to handle IFs. Nevertheless, their responses indicated a willingness to improve the current status, as long as sufficient resources are provided. The general population tended to support measures for handling IFs that go beyond those supported by investigators. Both investigators and the general population demanded more responsibility from PIs with physician licenses at medical institutions, although they were more lenient toward non-licensed PIs at non-medical institutions.

This study is empirical in its approach and is not intended to demonstrate clear norms. However, a large portion of the preliminary results obtained from this study was reflected in a proposal regarding approaches to IFs, which was subsequently included in the guidelines for national research projects. In this regard, the present study is of practical significance. We hope that the results of this study will be used as a reference, not only to help establish policies and guidelines in Japan and other countries but also to contribute to the development of neuroimaging research.

\begin{abstract}
Abbreviations
IF: Incidental finding; fMRI: Functional magnetic resonance imaging; MEXT: Ministry of Education, Culture, Sports, Science, and Technology; MRI: Magnetic resonance imaging; NIH: National Institutes of Health; PI: Principal investigator; SRPBS: Strategic Research Program for Brain Sciences.
\end{abstract}

\section{Competing interests}

The authors declare that they have no competing interests.

\section{Authors' contributions}

MF participated in the construction of the study design, data collection and analysis, manuscript drafting, and critical discussion. YH participated in the construction of the study design, data analysis, manuscript drafting, and critical discussion. ST participated in data collection and analysis and critical discussion. KT and EN participated in data analysis and critical discussion. AA participated in the construction of the study design, data analysis,

manuscript drafting, and critical discussion. All authors read and approved the final manuscript

\section{Authors' information}

MF (MS, MPH, PhD) is an Associate Professor of Uehiro Research Division for iPS Cell Ethics at the Center for iPS Cell Research and Application (CiRA), Kyoto University. She has been working as a clinical psychologist and researcher and conducting empirical studies of various bioethical issues such as transplantation, regenerative medicine, and neuroscience. $\mathrm{YH}(\mathrm{PhD})$ is an Associate Professor of Philosophy at Ritsumeikan University, Kyoto, Japan. He received a PhD in philosophy from Kyoto University, Kyoto, Japan. His research interests include ethical theories, theories of justice, neuroethics, and sport ethics. ST (PhD) is an Assistant Professor of Office for Promoting Medical Research at Showa University. He holds a PhD in Sociology from Tohoku University. KT (MPH) is a doctoral candidate and project researcher at UT-CBEL (University of Tokyo Center of Biomedical Ethics and Law). She received her $\mathrm{MPH}$ from the University of Tokyo. EN (PhD) studied at the Department of History and Philosophy of Science, University of Tokyo. He is presently an Assistant Professor at the Department of Biomedical Ethics at the University of Tokyo Graduate School of Medicine. His area is philosophy 
of memory, philosophy of science, and neuroethics. AA (MD, PhD) is Professor in the Department of Biomedical Ethics at the University of Tokyo Graduate School of Medicine and Director of UT-CBEL (University of Tokyo Center of Biomedical Ethics and Law). He also serves as Chair of the Ethics Committee at the University of Tokyo, Faculty of Medicine. His research interests include cross-cultural bioethics, informed consent, end-of-life issues, research ethics, bioethics policy, and clinical ethics.

\section{Acknowledgements}

This study is the result of "Research on resolving key issues in bioethics" carried out under the Strategic Research Program for Brain Sciences by the Ministry of Education, Culture, Sports, Science and Technology of Japan. The authors thank Mr. Katsumi Mori and Dr. Shizuko Takahashi at the University of Tokyo for their technical support on the manuscript.

\section{Author details}

${ }^{1}$ Uehiro Research Division for iPS Cell Ethics, Center for iPS Cell Research and Application (CiRA), Kyoto University, The Kyoto Technoscience Center \#3, 14, Yoshidakawara-cho, Sakyo-ku, Kyoto 606-8305, Japan. ${ }^{2}$ College of Letters, Human Studies Program, Ritsumeikan University, 56-1 Toji-in Kitamachi, Kita-ku, Kyoto 603-8577, Japan. ${ }^{3}$ Office for Promoting Medical Research, Showa University, 1-5-8 Hatanodai, Shinagawa-ku, Tokyo 142-8555, Japan. ${ }^{4}$ Department of Biomedical Ethics, The University of Tokyo Graduate School of Medicine, 7-3-1 Hongo, Bunkyo-ku, Tokyo 113-0033, Japan.

Received: 20 March 2014 Accepted: 24 September 2014

Published: 6 October 2014

\section{References}

1. Wolf SM, Lawrenz FP, Nelson CA, Kahn JP, Cho MK, Clayton EW, Fletcher JG, Georgieff MK, Hammerschmidt D, Hudson K, Illes J, Kapur V, Keane MA, Koenig BA, Leroy BS, McFarland EG, Paradise J, Parker LS, Terry SF, Van Ness $B$, Wilfond BS: Managing incidental findings in human subjects research: analysis and recommendations. J Law Med Ethics 2008, 36:219-248.

2. Illes J, Chin VN: Bridging philosophical and practical implications of incidental findings in brain research. J Law Med Ethics 2008, 36:298-304.

3. Illes J, Kirschen MP, Edwards E, Stanford LR, Bandettini P, Cho MK, Ford PJ, Glover GH, Kulynych J, Macklin R, Michael DB, Wolf SM: Ethics. Incidental findings in brain imaging research. Science 2006, 311:783-784.

4. Kirschen MP, Jaworska A, Illes J: Subjects' expectations in neuroimaging research. J Magn Reson Imaging 2006, 23:205-209.

5. Kumra S, Ashtari M, Anderson B, Cervellione KL, Kan L: Ethical and practical considerations in the management of incidental findings in pediatric MRI studies. J Am Acad Child Adolesc Psychiatry 2006, 45:1000-1006.

6. Wolf SM, Paradise J, Caga-anan C: The law of incidental findings in human subjects research: establishing researchers' duties. J Law Med Ethics 2008, 36:361-383.

7. Hayashi $Y$ : Ethical issues of handling incidental findings in neuroimaging research: clarifying and discussing some points. App/ Ethics 2010, 4:29-43 (In Japanese).

8. Lawrenz F, Sobotka S: Empirical analysis of current approaches to incidental findings. J Law Med Ethics 2008, 36:249-255.

9. Illes J, Kirschen MP, Karetsky K, Kelly M, Saha A, Desmond JE, Raffin TA, Glover GH, Atlas SW: Discovery and disclosure of incidental findings in neuroimaging research. J Magn Reson Imaging 2004, 20:743-747.

10. Royal JM, Peterson BS: The risks and benefits of searching for incidental findings in MRI research scans. J Law Med Ethics 2008, 36:305-314

11. Richardson HS: Incidental findings and ancillary-care obligations. J Law Med Ethics 2008, 36:256-270.

12. Miller FG, Mello MM, Joffe S: Incidental findings in human subjects research: what do investigators owe research participants? J Law Med Ethics 2008, 36:271-279.

13. Milstein AC: Research malpractice and the issue of incidental findings. J Law Med Ethics 2008, 36:356-360.

14. Illes J, Kirschen MP, Edwards E, Bandettini P, Cho MK, Ford PJ, Glover GH, Kulynych J, Macklin R, Michael DB, Wolf SM, Grabowski T, Seto B: Practical approaches to incidental findings in brain imaging research. Neurology 2008, 70:384-390.

15. Underwood E: When a brain scan bears bad news. Science 2012, 338:455
16. The National Core for Neuroethics: New Documentation for the Management of Incidental Findings. [http://neuroethics.med.ubc.ca/new-documentationfor-the-management-of-incidental-findings/]

17. The Ministry of Education, Culture, Sports, Science and Technology: The Strategic Research Program for Brain Sciences. [http://brainprogram.mext.go. jp/I (In Japanese).

18. The Japan Neuroscience Society: Guidelines for Ethical Issues of Non-Invasive Research on Human Brain Function. [http://www.jnss.org/wp-content/ uploads/2012/02/rinri.pdf] (In Japanese).

19. Sakura O, Fukushi T: Neuroethics: towards communicative relation among neuroscience and public. J Jpn Assoc Bioethics 2007, 17:18-27 (In Japanese).

20. Fujita M: Views among researchers, and the position of institutions, regarding the handling of incidental findings in research using brain images. In Brain Science Research and Ethics; Collection. Edited by Akabayashi A. Tokyo: The University of Tokyo; 2011:46-61. In Japanese.

21. Deslauriers C, Bell E, Palmour N, Pike B, Doyon J, Racine E: Perspectives of Canadian researchers on ethics review of neuroimaging research. $J$ Empir Res Hum Res Ethics 2010, 5:49-66.

22. Cooke $\mathrm{R}$, Peel $\mathrm{E}$, Shaw $\mathrm{RL}$, Senior $\mathrm{C}$ : The neuroimaging research process from the participants' perspective. Int J Psychophysiol 2007, 63:152-158.

23. Shaw RL, Senior C, Peel E, Cooke R, Donnelly LS: Ethical issues in neuroimaging health research: an IPA study with research participants. $J$ Health Psychol 2008, 13:1051-1059.

24. JMA Research Institute Inc. [http://www.jmar.co.jp/] (In Japanese)

25. Mamourian A: Incidental findings on research functional MR images: should we look. AJNR Am J Neuroradiol 2004, 25:520-522.

26. Booth TC, Jackson A, Wardlaw JM, Taylor SA, Waldman AD: Incidental findings found in 'healthy' volunteers during imaging performed for research: current legal and ethical implications. Br J Radiol 2010, 83:456-465.

27. Pickard JD, Gillard JH: Guidelines reduce the risk of brain-scan shock Nature 2005, 435:17

28. Ulmer S, Jensen UR, Jansen O, Mehdorn HM, Schaub J, Deuschl G, Siebner HR: Impact of incidental findings on neuroimaging research using functional MR imaging. AJNR Am J Neuroradiol 2009, 30:E55.

29. Shoemaker JM, Holdsworth MT, Aine C: A practical approach to incidental findings in neuroimaging research. Neurology 2011, 77:2123-2127.

30. Ross K: When volunteers are not healthy. EMBO Rep 2005, 6:1116-1119.

31. Hayashi $Y$ : Researcher's obligations to handle incidental findings and their implications. J Jpn Assoc Bioethics 2010, 21:22-29 (In Japanese).

32. Illes J, Rosen AC, Huang L, Goldstein RA, Raffin TA, Swan G, Atlas SW: Ethical consideration of incidental findings on adult brain MRI in research. Neurology 2004, 62:888-890.

33. Illes J: 'Pandora's box' of incidental findings in brain imaging research. Nat Clin Pract Neurol 2006, 2:60-61.

34. Appelbaum PS, Roth LH, Lidz C: The therapeutic misconception: informed consent in psychiatric research. Int J Law Psychiatry 1982, 5:319-329.

35. Appelbaum PS, Lidz CW: Twenty-five years of therapeutic misconception. Hastings Cent Rep 2008, 38:5-6.

36. Rangel EK: The management of incidental findings in neuro-imaging research: framework and recommendations. J Law Med Ethics 2010, 38:117-126.

37. Hayashi Y, Fujita M, Takashima K, Tashiro S, Akabayashi A: Recommendations. In The Management of Incidental Findings in Neuroimaging Research. Edited by Akabayashi A. Tokyo: The University of Tokyo; 2012:1-27. In Japanese.

\section{doi:10.1186/1478-4505-12-58}

Cite this article as: Fujita et al:: Handling incidental findings in neuroimaging research in Japan: current state of research facilities and attitudes of investigators and the general population. Health Research Policy and Systems 2014 12:58 